\title{
Avaliação de alunos de graduação em Medicina após treinamento na estratégia AIDPI
}

\author{
Edna Lúcia Santos de Souza ${ }^{1}$ \\ Maria Rosário Ribeiro Barretto ${ }^{2}$ \\ Geila Ribeiro Nuñez ${ }^{3}$ \\ Igor Fernando da Silva Carvalho ${ }^{3}$ \\ Leda Solano de Freitas Souza ${ }^{4}$
}

\begin{abstract}
Resumo
A Estratégia de Atenção Integrada às Doenças Prevalentes na Infância (AIDPI) foi adotada pelo Ministério da Saúde do Brasil em 1996, sendo adaptada de acordo com as características epidemiológicas às normas nacionais de atenção à criança. Desde 1997, a AIDPI vem progressivamente sendo implantada em diversos estados brasileiros. Algumas Universidades já incluem a AIDPI no curso médico. Em dezembro de 2002, foi iniciado treinamento na estratégia AIDPI para alunos de graduação em Medicina da UFBA. O presente estudo teve como objetivo avaliar o grau de mudança no conhecimento e nas atitudes de estudantes de Medicina após treinamento na estratégia AIDPI. Foi realizado um estudo longitudinal prospectivo no período de dezembro de 2002 a junho de 2003. Catorze alunos constituíram 2 coortes que receberam treinamento na estratégia AIDPI entre dezembro de 2002 e junho de 2003 . No ponto zero do acompanhamento de cada coorte, foi realizado um teste com questôes referentes a temas de conhecimentos básicos em pediatria, que também fazem parte do programa do curso de treinamento m AIDPI. O teste foi repetido nos desfechos das coortes. Foram utilizados a Prova dos Sinais e o teste de Mcnemar para análise estatística das variáveis estudadas, utilizando-se o software SPSS. Após o treinamento, houve mudanças estatisticamente significativas no conhecimento dos estudantes sobre os principais sinais de doença grave, de pneumonia, de pneumonia grave, de desnutrição grave, de desidratação e do uso apropriado de vacinas. O conhecimento dos estudantes sobre a taxa de mortalidade infantil e causas de óbitos em crianças também se modificou de forma estatisticamente significativa. Houve significativa melhoria nas habilidades de orientação das técnicas adequadas para amamentação exclusiva e para introdução de outros alimentos no desmame. Os resultados demonstraram que o treinamento na estratégia AIDPI gerou modificaçóes estatisticamente significativas nos conhecimentos e atitudes dos alunos avaliados.
\end{abstract}

Palavras chave: AIDPI; estudantes de Medicina - treinamento.

\section{INTRODUÇÃO}

Diante da elevada morbimortalidade por doenças preveníveis ou tratáveis em crianças menores de 5 anos, em países em desenvolvimento, e da necessidade de se atingir a meta de redução da mortalidade na infância proposta pelos países da Região das Américas para o ano de 2000, a Organização Pan-Americana de Saúde/Organização Mundial de Saúde (OPAS/

\footnotetext{
${ }^{1}$ Professora Assistente do Departamento de Pediatria da Faculdade de Medicina da UFBA; Coordenadora da implantação do AIDPI na UFBA. Salvador - BA

2 Médica da Secretaria da Saúde do Estado da Bahia. Salvador - BA

${ }^{3}$ Acadêmico de Medicina da Universidade Federal da Bahia. Salvador - BA

4 Professora Adjunto do Departamento de Pediatria da Faculdade de Medicina da UFBA. Salvador - BA

Correspondência para / Correspondence to:

Edna Lúcia Santos de Souza

Centro Pediátrico Prof. Hosannah de Oliveira (CPPHO).

Rua Pe. Feijó, s/n - Canela.

40.110-170 Salvador- Bahia- Brasil.

E-mail: ednalu@ufba.br.
} 
OMS), em conjunto com o Fundo das Nações Unidas, (UNICEF) elaborou a Estratégia de Atenção Integrada as Doenças Prevalentes na Infância (AIDPI), incorporando estratégias individuais já existentes para controle de doenças e problemas específicos de saúde. ${ }^{1,2}$

A estratégia AIDPI tem por finalidade promover uma rápida e significativa redução da mortalidade na infância. Caracteriza-se pela consideração simultânea e integrada do conjunto de doenças de maior prevalência na infância, ao invés da abordagem tradicional que avalia cada doença isoladamente. Enfoca diretamente as doenças prevalentes: infecções respiratórias agudas, diarréia, malária, desnutrição e anemia (que estão muitas vezes associadas) e os agravos resultantes do desmame precoce e da baixa cobertura vacinal. Devido às suas características, a utilização da estratégia evita que se percam oportunidades de detecção de problemas. ${ }^{3,4}$

A estratégia AIDPI foi adotada pelo Ministério da Saúde do Brasil (MS) em 1996, sendo adaptada de acordo com as características epidemiológicas às normas nacionais de atenção à criança. ${ }^{4}$

Desde 1997, a AIDPI vem progressivamente sendo implantada em diversos estados brasileiros, tanto no âmbito das secretarias de saúde estaduais e municipais, quanto das instituições de ensino superior. As universidades foram envolvidas pelo MS a partir de 1998. Essas instituições de ensino têm um papel relevante na formação de médicos e enfermeiros com treinamento nessa estratégia. ${ }^{4}$

Atualmente, diversas Universidades brasileiras, a exemplo das Universidades Federais de Sergipe e Paraíba e da Universidade de São Paulo, já incluem a AIDPI no currículo da graduação em Medicina.

$\mathrm{Na}$ Faculdade de Medicina da Bahia, as ações em direção à implantação da AIDPI no currículo médico consistiram da participação isolada de poucos docentes em treinamentos promovidos pelo Ministério da Saúde, em 1996 e em 1999. A partir daí, algumas atividades foram realizadas no âmbito da Universidade Federal da Bahia (UFBA), visando à sensibilização e ao treinamento mais abrangente de profissionais: em 2000, curso para docentes do Departamento de Pediatria; em 2001, um seminário de sensibilização para todos os profissionais de saúde do Centro Pediátrico Prof. Hosannah de Oliveira (CPPHO), um curso de treinamento em AIDPI para médicos e enfermeiras do $\mathrm{CPPHO}$ e um curso para residentes em Pediatria, no qual também foi treinada uma professora do Departamento de Pediatria da FAMEB. No total, até o momento, 11 docentes de Pediatria foram treinados, incluindo 5 professores substitutos, que já não fazem parte do Departamento. Em 2001, foi apresentado um projeto para implantação da estratégia no setor de Pronto Atendimento do $\mathrm{CPPHO}$, que, no entanto, foi inviabilizado por diversas circunstâncias. O CPPHO é um centro hospitalar e ambulatorial administrado pela UFBA, que recebe os alunos do curso de Medicina e médicos residentes em pediatria, bem como estudantes de outras áreas, como enfermagem, nutrição e fisioterapia. Em 2002, o Departamento de Pediatria aprovou a implantação da estratégia no internato em pediatria, tendo sido iniciado o treinamento de estudantes em dezembro.

O curso de Medicina da UFBA tem duração de 12 semestres e recebe 80 alunos semestralmente. No $6^{\circ}$ semestre, os alunos têm o primeiro contato com a disciplina de Pediatria (Pediatria Preventiva e Social), com carga horária de 120 horas, quando fazem atendimento ambulatorial e participam de sessões interativas. Posteriormente, os estudantes só voltam a cursar pediatria no internato rotatório, nos 4 últimos semestres do curso. Durante o internato, o Departamento de Pediatria recebe 40 alunos a cada 3 meses, para estágios de 10 semanas no $9^{\circ}$ ou no $10^{\circ}$ semestre, e de 12 semanas no $11^{\circ}$ ou no $12^{\circ}$ semestre. Supõe-se que a introdução da Estratégia AIDPI no curso médico deverá beneficiar a formação dos estudantes, representando um esforço para equilibrar parcialmente a forte tendência hospitalocêntrica que ainda caracteriza o curso de Medicina da UFBA. É fundamental saber se o curso que está sendo realizado está tendo impacto positivo nos estudantes. Assim, o objetivo desta investigação foi avaliar o grau de mudança no conhecimento e nas atitudes dos estudantes após o treinamento.

\section{MATERIAL E MÉTODOS}

Foi realizado um estudo longitudinal prospectivo no período de dezembro de 2002 a 
junho de 2003. Os alunos constituíram 2 coortes: 7 estudantes do 11을 semestre e 7 estudantes do 9 o semestre, que receberam treinamento, respectivamente, entre dezembro de 2002 e janeiro de 2003, e de abril a junho de 2003.

Foi apresentada uma visão geral da estratégia AIDPI aos estudantes do internato, que estavam, então, iniciando o seu estágio em pediatria, sendo realizado um sorteio para escolha de um máximo de 7 alunos, desde que havia apenas um docente para supervisão. $\mathrm{O}$ curso teve duração de 8 semanas, com carga horária total de 40 horas. O treinamento consistiu em sessões de estudo dirigido, utilizando-se o módulo da AIDPI adaptado para a graduação ${ }^{5}$ e atividades práticas, que ocorreram no setor de Pronto Atendimento do CPPHO. Embora tivesse como enfoque principal o treinamento em AIDPI, o curso não se limitou a discutir a estratégia. Os alunos foram estimulados a realizar pesquisas sobre temas correlacionados e trazerem os resultados do estudo para discussão em sessões posteriores. As atividades em sala de aula tiveram supervisão do autor principal, sendo que as atividades práticas contaram também com a supervisão de um dos demais autores.

No ponto zero do acompanhamento de cada coorte, foi realizado um teste (QUADRO1), com questōes referentes a temas básicos em pediatria, abordados na Disciplina de Pediatria Preventiva e Social no 6o semestre, em aulas práticas e (ou) sessóes interativas. Os mesmos temas fazem parte do programa do curso de treinamento em AIDPI. O teste foi repetido nos desfechos das coortes. Solicitou-se também que os estudantes preenchessem um questionário de avaliação do curso ao final do treinamento. O estudo foi aprovado pelo Comitê de Ética em Pesquisas Humanas da Maternidade Climério de Oliveira.

\section{ANÁLISE DOS DADOS}

Os questionários respondidos pelos alunos foram confrontados com um padrão-ouro, baseado no programa AIDPI. A avaliação não

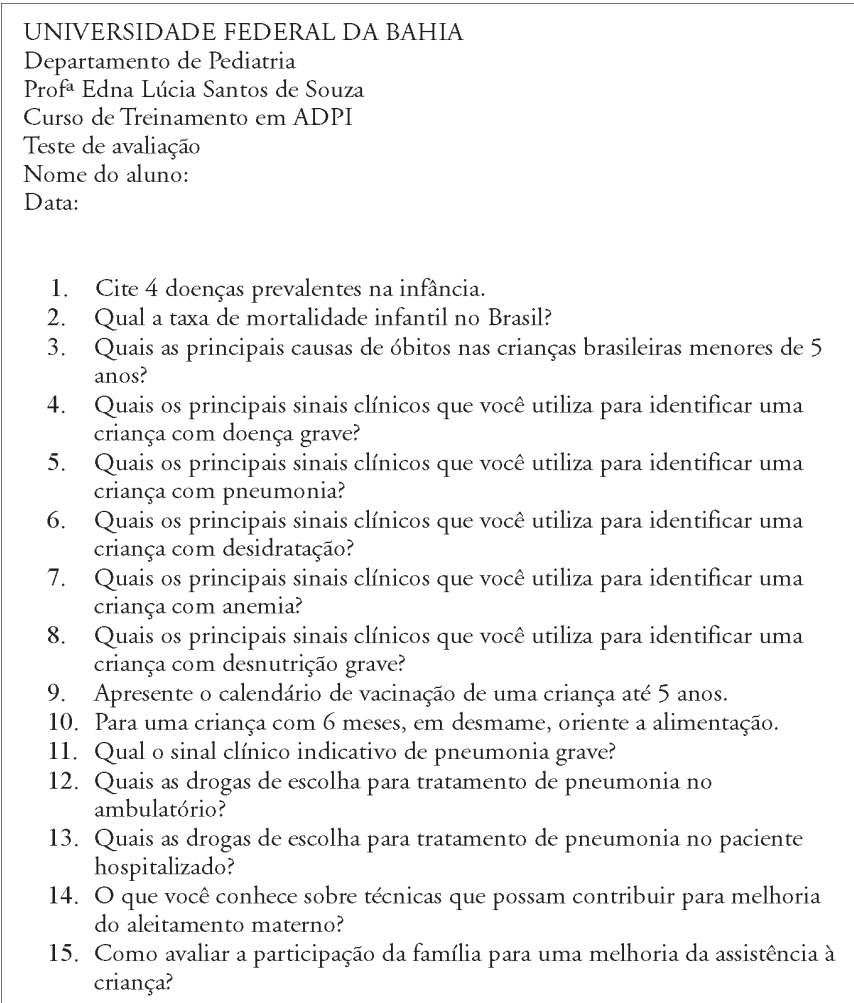

Quadro 1 - Teste de avaliação dos estudantes, aplicado antes e após o treinamento na AIDPI 
foi "cega" quanto ao conhecimento de quais eram os questionários prévios ou posteriores ao treinamento. As questões foram agrupadas em 2 categorias, conforme o modo como foram computados os acertos:

I. Questôes objetivas com respostas incluindo 1 ou mais itens (questões $1,34,5,6$, $7,8,11,12$ e 13): as questôes foram classificadas em corretas (iguais ao padrão-ouro), aceitáveis (não contidas no padrão-ouro, porém ainda assim corretas) e incorretas; a cada resposta correta foi atribuído um ponto positivo, à resposta aceitável foi atribuído 0 ponto e, a cada resposta incorreta, atribuiu-se 1 ponto negativo; a pontuação final da questão foi a soma dos pontos, sendo a nota mínima zero. A questão 9 foi interpretada individualmente, sendo computado 1 ponto para quem tivesse um número de respostas corretas igual ou superior a 14 e foi pontuado como zero o número de acertos entre 1 e 13.

II. Questôes com importante componente subjetivo (questôes 10,14 e 15): cada uma das respostas foi avaliada por 2 pediatras treinados em AIDPI, que as classificaram em respostas satisfatórias, parcialmente satisfatórias ou insatisfatórias.

A questão 2 não foi incluída em nenhuma das categorias acima: as respostas foram classificadas como certas ou erradas.

A avaliação das respostas permitiu avaliar a aquisição do conhecimento pelos alunos durante o treinamento; adicionalmente, as questões subjetivas possibilitaram vislumbrar indícios de mudanças de atitude dos estudantes em relação a alguns aspectos do atendimento à criança.

Foi feita uma análise estatística das variáveis estudadas nos testes no início e no desfecho das coortes, utilizando-se o software SPSS (Statistical Packge for Social Sciences) versão 10.0 for Windows. Utilizou-se a Prova dos Sinais para avaliar as variáveis $1,3,4,5,6,7,8,9$ e 10 . O teste de Mcnemar foi utilizado para as variáveis 2 e 13. Foi considerado nível de significância estatística os valores de "p" igual ou inferiores a 0,05 . As questōes 12,14 e 15 tiveram apenas análise descritiva.

\section{RESULTADOS}

Os resultados estão disponíveis nas Tabelas 1, 2 e 3, e nas Figuras, 1, 2, 3 e 4.

Entre os alunos avaliados, $50 \%$ obtiveram acerto total da questão "quais as doenças mais prevalentes da infância?" na primeira avaliação, contrapondo-se a $78,6 \%$ da amostra na segunda avaliação, diferença estatisticamente não significante $(p=0,375)$. No primeiro teste, apenas $7,1 \%$ dos estudantes responderam corretamente sobre qual a taxa de mortalidade infantil no Brasil, enquanto, no teste 2 , a frequiência de acertos aumentou para 92,9\% ( $\mathrm{p}=0,021)$. Cerca de $29 \%$ dos estudantes, no teste 1 , e $85,7 \%$, no teste 2 , citaram corretamente pelo menos duas causas de óbito em crianças menores de 5 anos ( $\mathrm{p}=0,001$, figura 1$)$. Quanto aos sinais clínicos para identificar criança com doença grave, nenhum aluno, no primeiro teste, citou qualquer sinal clínico correto; no segundo teste, 78,6\% dos estudantes da amostra acertaram completamente a questão $(\mathrm{p}<0,001)$. Cinqüenta por cento dos estudantes acertaram sobre o sinal clínico para identificar uma criança com pneumonia na primeira avaliação, freqüência que ascendeu para $100 \%$ da amostra quando avaliados pela segunda vez $(\mathrm{p}=0,016)$. Já os sinais clínicos para identificar uma criança com desidratação (figura 2) não foram referidos corretamente por nenhum aluno no teste 1 , contrapondo-se a $42,9 \%$ de acerto total no teste 2 $(\mathrm{p}<0,001)$. Não houve diferença estatisticamente significante $(\mathrm{p}=0,5)$ ao se compararem os índices de acertos da questão "quais os sinais clínicos utilizados para identificar uma criança com anemia?" no teste $1(85,7 \%)$ e no teste 2 $(100 \%)$. Ao serem avaliados pela primeira vez, $21,4 \%$ dos estudantes responderam corretamente sobre os sinais clínicos para identificar uma criança com desnutrição grave (figura 3); na segunda avaliação, a freqüência de acertos aumentou para $78,6 \%(\mathrm{p}=0,004)$. No primeiro teste, $28,6 \%$ dos alunos referiram corretamente 14 ou mais indicações de vacinas para crianças menores de 5 anos, de acordo com o calendário vacinal do Ministério da Saúde (gráfico 4) e, no teste 2, o número de alunos que teve número de acertos igual ou maior aumentou para $85,7 \%$ 
Tabela 1 - Questōes objetivas: Freqüência das pontuações obtidas pelos estudantesnos testes 1 e 2 das questôes 1, 3-9 e 11-13, Salvador, 2003

\begin{tabular}{|c|c|c|c|c|c|c|c|}
\hline \multicolumn{2}{|c|}{ Pontuação obtida } & \multirow{2}{*}{$\begin{array}{c}0 \\
0(0 \%)\end{array}$} & \multirow{2}{*}{$\begin{array}{c}1 \\
0(0 \%)\end{array}$} & \multirow{2}{*}{$\begin{array}{c}2 \\
0(0 \%)\end{array}$} & \multirow{2}{*}{$\begin{array}{c}3 \\
7(50 \%)\end{array}$} & \multirow{2}{*}{$\begin{array}{c}4 \\
7(50 \%)\end{array}$} & \multirow{2}{*}{$\begin{array}{c}\begin{array}{c}\text { Năo } \\
\text { respondeu }\end{array} \\
0(0 \%)\end{array}$} \\
\hline Questão 1 & Teste 1 & & & & & & \\
\hline & Teste 2 & $0(0 \%)$ & $0(0 \%)$ & $1(7,1 \%)$ & $2(14,3 \%)$ & $11(78,6 \%)$ & $0(0 \%)$ \\
\hline \multirow[t]{2}{*}{ Questão 3} & Teste 1 & $3(21,4 \%)$ & $7(50 \%)$ & $4(28,6 \%)$ & $0(0 \%)$ & $0(0 \%)$ & $0(0 \%)$ \\
\hline & Teste 2 & $0(0 \%)$ & $2(14,3 \%)$ & $6(42,9 \%)$ & $3(21,4 \%)$ & $3(21,4 \%)$ & $0(0 \%)$ \\
\hline \multirow[t]{2}{*}{ Questão 4} & Teste 1 & $6(42,9 \%)$ & $6(42,9 \%)$ & $0(0 \%)$ & $0(0 \%)$ & $0(0 \%)$ & $2(14,3 \%)$ \\
\hline & Teste 2 & $0(0 \%)$ & $0(0 \%)$ & $1(7,1 \%)$ & $2(14,3 \%)$ & $11(78,6 \%)$ & $0(0 \%)$ \\
\hline \multirow[t]{2}{*}{ Questăo 5} & Teste 1 & $7(50 \%)$ & $7(50 \%)$ & $x^{1}$ & $\mathrm{x}$ & $\mathrm{x}$ & $0(0 \%)$ \\
\hline & Teste 2 & $0(0 \%)$ & $14(100 \%)$ & $\mathrm{x}$ & $\mathrm{x}$ & $\mathrm{x}$ & $0(0 \%)$ \\
\hline \multirow[t]{2}{*}{ Questão 6} & Teste 1 & $9(64,3 \%)$ & $3(21,4 \%)$ & $1(7,1 \%)$ & $1(7,1 \%)$ & $0(0 \%)$ & $0(0 \%)$ \\
\hline & Teste 2 & $0(0 \%)$ & $0 \quad(0 \%)$ & $2(14,3 \%)$ & $6(42,9 \%)$ & $6(42,9 \%)$ & $0(0 \%)$ \\
\hline \multirow[t]{2}{*}{ Questão 7} & Teste 1 & $2(14,3 \%)$ & $12(85,7 \%)$ & $\mathrm{x}$ & $\mathrm{x}$ & $\mathrm{x}$ & $0(0 \%)$ \\
\hline & Teste 2 & $0(0 \%)$ & $14(100 \%)$ & $\mathrm{x}$ & $\mathrm{x}$ & $\mathrm{x}$ & $0(0 \%)$ \\
\hline \multirow[t]{2}{*}{ Questão 8} & Teste 1 & $6(42,9 \%)$ & $5(35,7 \%)$ & $3(21,4 \%)$ & $\mathrm{x}$ & $\mathrm{x}$ & $0(0 \%)$ \\
\hline & Teste 2 & $0(0 \%)$ & $3(21,4 \%)$ & $11(78,6 \%)$ & $\mathrm{x}$ & $\mathrm{x}$ & $0(0 \%)$ \\
\hline \multirow[t]{2}{*}{ Questão 9} & Teste 1 & $8(57,1 \%)$ & $4(28,6 \%)$ & $\mathrm{x}$ & $\mathrm{x}$ & $\mathrm{x}$ & $2(14,3 \%)$ \\
\hline & Teste 2 & $1(7,1 \%)$ & $12(85,7 \%)$ & $\mathrm{x}$ & $\mathrm{x}$ & $\mathrm{x}$ & $1(7,1 \%)$ \\
\hline \multirow[t]{2}{*}{ Questãol1 } & Teste 1 & $14(100 \%)$ & $0(0 \%)$ & $\mathrm{x}$ & $\mathrm{x}$ & $\mathrm{x}$ & $0(0 \%)$ \\
\hline & Teste 2 & $0(0 \%)$ & $14(100 \%)$ & $\mathrm{x}$ & $\mathrm{x}$ & $\mathrm{x}$ & $0(0 \%)$ \\
\hline \multirow[t]{2}{*}{ Questăo 12} & Teste 1 & $2(14,3 \%)$ & $12(85,7 \%)$ & $\mathrm{x}$ & $\mathrm{x}$ & $\mathrm{x}$ & $0(0 \%)$ \\
\hline & Teste 2 & $0(0 \%)$ & $14(100 \%)$ & $\mathrm{x}$ & $\mathrm{x}$ & $\mathrm{x}$ & $0(0 \%)$ \\
\hline \multirow[t]{2}{*}{ Questão 13} & Teste 1 & $2(14,3 \%)$ & $12(85,7 \%)$ & $\mathrm{x}$ & $\mathrm{x}$ & $\mathrm{x}$ & $0(0 \%)$ \\
\hline & Teste 2 & $2(14,3 \%)$ & $12(85,7 \%)$ & $x$ & $x$ & $x$ & $0(0 \%)$ \\
\hline
\end{tabular}

Tabela 2 - Questões subjetivas: Freqüências dos tipos de avaliações das respostas das questões 10 e 15 nos testes 1 e 2, Salvador, 2003.

\begin{tabular}{lccrrrr}
\hline \multicolumn{1}{c}{ Tipos de respostas } & \multicolumn{2}{c}{ Questáo 10 } & \multicolumn{2}{c}{ Questão 14 } & \multicolumn{2}{c}{ Questão 15 } \\
\hline & \multicolumn{1}{c}{ Teste 1 } & \multicolumn{1}{c}{ Teste 2 } & \multicolumn{1}{c}{ Teste 1 } & \multicolumn{1}{c}{ Teste 2 } & Teste 1 & Teste 2 \\
Resposta satisfatória & $4(28,6 \%)$ & $10(71,4 \%)$ & $5(35,7 \%)$ & $14(100 \%)$ & $0(0 \%)$ & $4(57,1 \%)$ \\
Resposta parcialmente & $8(57,1 \%)$ & $4(28,6 \%)$ & $6(42,9 \%)$ & $0(0 \%)$ & $2(28,6 \%)$ & $2(28,6 \%)$ \\
satisfatória & $1(7,1 \%)$ & $0(0 \%)$ & $1(7,1)$ & $0(0 \%)$ & $0(0 \%)$ & $0(0 \%)$ \\
Resposta insatisfatória & $1(7,1 \%)$ & $0(0 \%)$ & $2(14,3 \%)$ & $0(0 \%)$ & $5(71,4 \%)$ & $1(14,3 \%)$ \\
Não respondeu & & & & &
\end{tabular}

Tabela 3- Questão 2 :Freqüência do número de respostas corretas, incorretas e ausentes, dos estudantes na $2^{\text {a }}$ questão nos testes 1 e 2, Salvador, 2003.

\begin{tabular}{lrr}
\hline Tipo de acerto & \multicolumn{2}{c}{ Questão 2 } \\
\hline & Teste 1 & \multicolumn{1}{c}{ Teste 2 } \\
Resposta correta & $1(7,1 \%)$ & $13(92,9 \%)$ \\
Resposta incorreta & $9(64,3 \%)$ & $1(7,1 \%)$ \\
Não respondeu & $4(28,6)$ & $0(0,0 \%)$ \\
\hline
\end{tabular}

$(\mathrm{p}=0,012)$. Nenhum dos alunos soube informar sobre o sinal clínico de pneumonia grave na primeira avaliação, enquanto todos acertaram na avaliação 2. Quanto à droga de escolha para tratamento de pneumonia em nível ambulatorial, 85,7\% dos alunos responderam corretamente no primeiro teste, enquanto, $100 \%$ o fizeram no teste 2 . Já o tratamento em âmbito hospitalar foi citado corretamente por $85,7 \%$ dos estudantes em ambos os testes. 


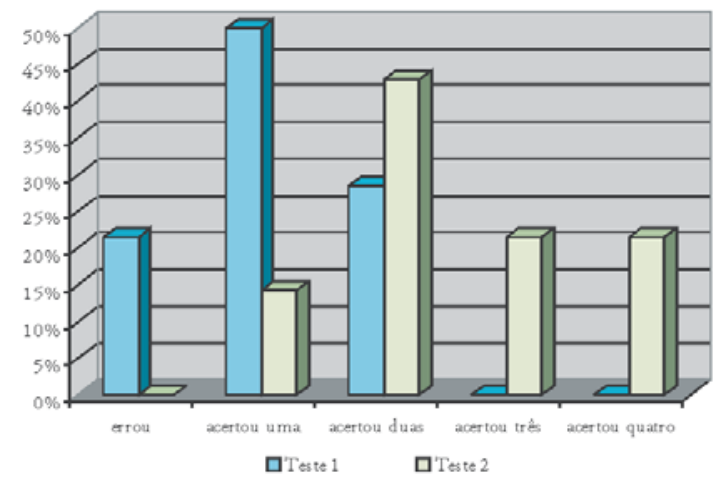

Figura 1 - Causas principais de mortalidade : Comparação dos índices de acertos entre os dois testes da $3^{a}$ questão (Quais as principais causas de óbitos nas crianças brasileiras menores de 5 anos?). $\mathrm{p}<0,05$

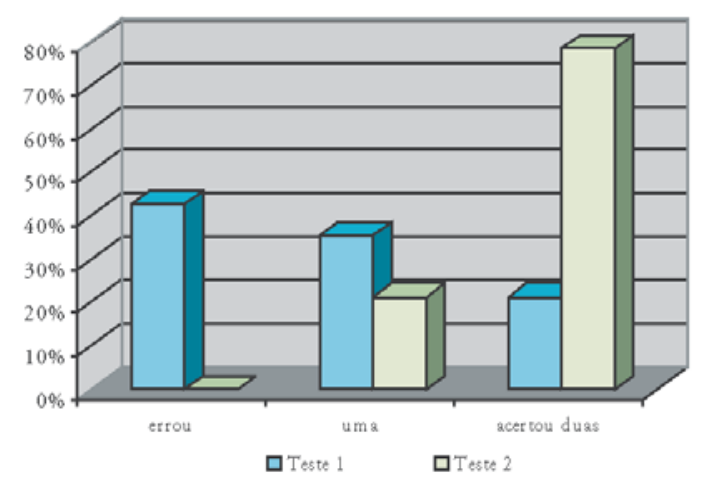

Figura 3 -Sinais de desnutrição grave: comparação dos índices de acertos entre os dois testes da $8^{a}$ questão (Quais os principais sinais clínicos que você utiliza para identificar uma criança com desnutrição grave?). $\mathrm{p}<0,05$

As questôes que permitiam avaliar subjetivamente a atitude do estudante diante do paciente mostraram que $28,6 \%$ e $71,4 \%$ dos alunos, nos testes 1 e 2 , respectivamente, orientaram satisfatoriamente a alimentação de uma criança de 6 meses em desmame ( $p>0,05)$. Ao serem questionados pela primeira vez sobre as técnicas para assegurar uma melhoria do aleitamento materno, $35,7 \%$ dos estudantes no teste 1 obtiveram uma resposta satisfatória, enquanto que, no teste 2 , essa freqüência foi de $100 \%$. A última questão da avaliação - "como se avalia a participação da família para melhoria da assistência à criança?" - foi respondida apenas pelos alunos da primeira coorte. $\mathrm{Na}$ primeira avaliação, $71,4 \%$ não responderam e $28,6 \%$ o fizeram parcialmente; na segunda avaliação, $57,1 \%$

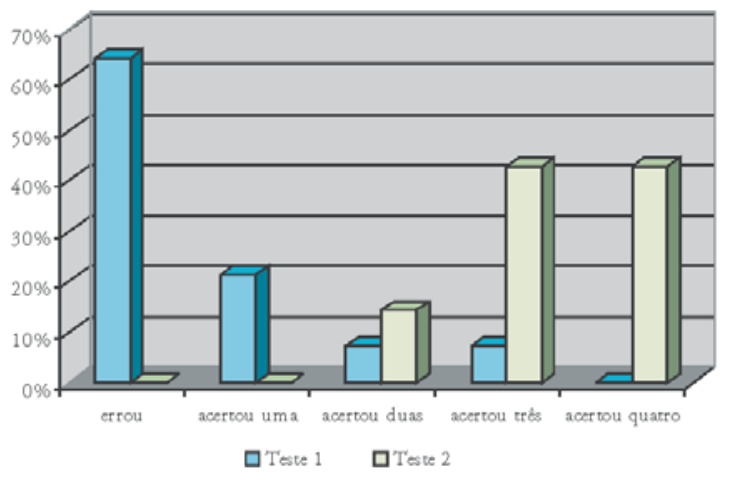

Figura 2 - Sinais de Desidratação: Comparação dos índices de acertos entre os dois testes da $6^{a}$ questão (quais os principais sinais clínicos que você utiliza para identificar uma criança com desidratação ? ). p < 0,05

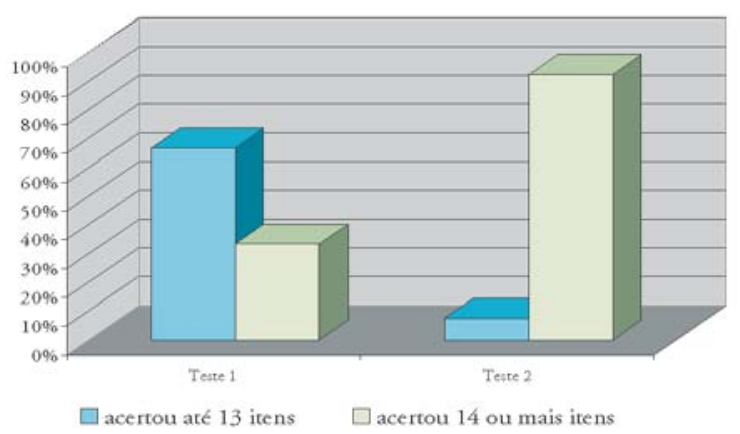

Figura 4 - Calendário Vacinal: Comparação dos índices de acertos entre os dois testes da $9^{a}$ questão (Apresente o calendário de vacinação de uma criança até os 5 anos). $\mathrm{p}<0,05$

deram respostas satisfatórias, $28,6 \%$ deram respostas parciais e $14,3 \%$ não responderam.

\section{DISCUSSÃO}

Uma análise do atual currículo da FAMEB revela algumas distorçōes: persistência da prática de ensino no Hospital Universitário (centralizando os recursos técnicos científicos mais avançados para a investigação biológica) e o desligamento das atividades assistenciais comunitárias; a falta de integração entre as disciplinas; a ocorrência de uma lacuna de 2 semestres, em que não há qualquer atividade na área de Pediatria, entre o 60 semestre, quando os estudantes cursam a Disciplina Pediatria 
Preventiva e Social, e os 4 últimos semestres do curso, quando se desenvolve o Internato. A Pediatria corresponde a uma das cinco grandes áreas da Medicina, nas quais os alunos realizam estágios durante o internato.

Este estudo, surpreendentemente, mostrou que cerca de $93 \%$ dos alunos avaliados, que cursam o $11^{\circ}$ semestre, prestes a concluir o curso médico, e também alunos do 9o semestre em Medicina, desconheciam a taxa de mortalidade infantil brasileira antes do treinamento. Sabendo-se da importância desse índice como expressão do desenvolvimento socioeconômico de um país (6), espera-se que o médico tenha conhecimento dessa taxa. Após o treinamento, 93\% dos alunos responderam de forma correta a essa questão. É fundamental que os médicos conheçam o calendário vacinal da criança, mas, antes do treinamento, mais da metade dos estudantes avaliados desconhecia as principais vacinas a serem administradas até os 5 anos, apesar de esse tema ser amplamente debatido durante o curso em vários momentos. Houve uma mudança estatisticamente significativa após o treinamento. Conhecimentos tão simples, mas essenciais para uma prática médica adequada não foram assimilados ao longo do curso médico. Chamaram atenção as dificuldades apresentadas pelos estudantes, antes do treinamento, em responder a última questão, que tratou do papel da família na assistência à criança. Percebeu-se, após o curso, uma mudança marcante na atitude do aluno, com respostas claras, marcadas pela compreensão da vital importância da família e do contexto socioeconômico na saúde da criança, bem como da necessidade da verificação do entendimento, pela família, das informações prestadas pelo profissional. Este estudo reafirmou a necessidade de refletir sobre a eficácia pedagógica do Curso Médico, bem como sobre a importância que o estudante credita à aquisição de conhecimentos básicos.

Cabe ressaltar que os dois grupos não foram homogêneos, desde que a primeira coorte foi composta de alunos do $11^{\circ}$ semestre, enquanto a segunda foi constituída de alunos do 9․ O primeiro grupo de alunos, por estar em fase mais acelerada do Curso Médico, demonstrou um bom grau de conhecimento em temas variados, enriquecendo as discussões nas sessões interativas, durante o treinamento.

Este estudo tem limitações, em decorrência da amostra pequena e do instrumento utilizado (questionário), imperfeito para mensurar mudanças subjetivas e mudanças de atitudes. As coortes foram analisadas em conjunto, por causa do pequeno número de alunos. Apesar das limitações referidas, os resultados revelaram mudanças significativas nos conhecimentos, após o treinamento; observouse também uma uniformização das respostas dos alunos, no segundo teste, o que indica a assimilação das informações. Embora a AIDPI aborde temas básicos, utilizando um conteúdo mínimo de informações, o treinamento utiliza uma sistematização que pode contribuir muito para a sedimentação dos conhecimentos.

O questionário de avaliação do curso pelos alunos revelou que $100 \%$ deles consideraram o curso relevante e com uma contribuição significativa na sua formação. $O$ primeiro grupo considerou que o treinamento deveria ocorrer mais precocemente. Essa reflexão determinou a antecipação do treinamento para $9^{\circ}$ semestre, no segundo grupo. Os estudantes e o autor principal perceberam que o conteúdo da prática era limitado; assim, foram planejadas modificações programáticas para os grupos de alunos a serem treinados no futuro.

A análise dos resultados permite concluir sobre a relevância em treinar os estudantes na estratégia AIDPI e desperta algumas reflexões: "Qual o momento ideal para a realização do treinamento?", "Qual a melhor forma de realização do treinamento: em reuniōes semanais ou como curso intensivo?". Acreditamos que a realização mais precoce, no $6^{\circ}$ semestre, e a ampliação de atividades práticas acarretem ganho de eficiência.

Pode-se concluir que o treinamento na estratégia foi válido para os grupos avaliados, gerando modificações significativas nos conhecimentos e atitudes dos alunos. Sugere-se que esse treinamento seja ampliado para todos os estudantes do Curso Médico, e incorporado ao conteúdo programático da Pediatria.

R. Ci. méd. biol., Salvador, v. 4, n. 1, p. 7-14, jan./abr. 2005 


\title{
Evaluation of the effect of IMCI training on medical students
}

\begin{abstract}
The WHO strategy of Integrated Management of Childhood Illnesses (IMCI) was adopted in 1996 by the Ministry of Health of Brazil, as a program of public health, in accordance with the national rules related to children care. Since 1997, the program has been set up in several Brazilian states, and has been included in the medical course in some universities. To evaluate the changes in medical students' attitudes and knowledge about pediatric primary care, after a workshop on IMCI. Cohort prospective study: fourteen medical students were enrolled and trained between December 2002 and June 2003. The students answered a questionnaire about pediatric primary care, before and after the training period. The first and second answered questionnaires were compared statistically, using the signal and McNemar tests. SPSS software was used for data entry and statistical analysis. There were significant changes in the students' knowledge about clinical signs of severe disease, of pneumonia, of severe pneumonia, of severe undernutrition, of dehydration and use of appropriate vaccines. There were significant changes in the students knowledge about infantile mortality and mortality causes in children. There were significant improvements in the abilities to correctly indicate substitute food for breast milk during and after weaning, and to teach mothers how to breastfeed. The medical student attitudes and knowledge about prevalent pediatric illnesses and about children's primary care have been significantly modified by the training in IMCI.
\end{abstract}

Keywords: IMCI; medical students-training.

\section{REFERÊNCIAS}

1 BENGUIGUI. Y. Atenção Integrada às Doenças Prevalentes na Infância. In: et al. (Ed.) Ações de saúde materno infantil a nível local segundo as metas da Cúpula Mundial em Favor da Infância. Washington, DC: Organização Panamericana da Saúde, 1997. p.171-182.

2. ORGANIZAÇÃO PANAMERICANA DA SAÚDE. Atenção Integrada às Doenças Prevalentes na Infância- AIDPI 2002. Washington, DC, 1999.

3. BRASIL. Ministério da Saúde. Secretaria de Política de Saúde. Área da Saúde da Criança. AIDPI - Atenção Integrada às Doenças Prevalentes na Infância: curso de capacitação: Módulo 1 - Introdução. 2.ed.rev. Brasília, DF, 2003.

4. ORGANIZAÇÃO PANAMERICANA DA SAÚDE. Atenção Integrada às Doenças
Prevalentes na Infância- AIDPI: Avaliação nas Unidades de Saúde. Brasília, DF, 2002.

5. ORGANIZAÇÃO PANAMERICANA DA SAÚDE. AIDPI para o ensino médico. Brasília, DF, 2002.

6. LEONE, C.; ALCANTARA, P. Etiologia geral da morbidade e mortalidade da criança. In: MARCONDES, E. et al. Pediatria básica. São Paulo: Sarvier, 2002. p.14-23.

7. FUNDAÇÃO NACIONAL DE SAÚDE. Centro Nacional de Epidemiologia-CENEPI. Brasília, DF, 1999.

8. BRICKS, L.F.; OSELKA, G.W. Imunizaçōes. In: MARCONDES, E. et al. Pediatria básica. São Paulo: Sarvier, 2002. p.102-113.

9. BRASIL. Ministério da Saúde. Guia alimentar para as crianças menores de dois anos. Basília, DF, 2002.

\section{Agradecimentos}

Aos estudantes de Medicina, por aceitarem colaborar conosco nesta pesquisa, e ao Prof. Maurício Cardeal, por nos auxiliar na análise estatística dos resultados. 